\title{
Glassy carbon microelectrodes minimize induced voltages, mechanical vibrations, and artifacts in magnetic resonance imaging
}

\author{
Surabhi Nimbalkar ${ }^{1,2}$, Erwin Fuhrer (10) 3 , Pedro Silva ${ }^{3}$, Tri Nguyen ${ }^{1,2}$, Martin Sereno $\mathbb{E}^{4}$, Sam Kassegne ${ }^{1,2}$ and
} Jan Korvink (i) ${ }^{3}$

\begin{abstract}
The recent introduction of glassy carbon (GC) microstructures supported on flexible polymeric substrates has motivated the adoption of GC in a variety of implantable and wearable devices. Neural probes such as electrocorticography and penetrating shanks with GC microelectrode arrays used for neural signal recording and electrical stimulation are among the first beneficiaries of this technology. With the expected proliferation of these neural probes and potential clinical adoption, the magnetic resonance imaging (MRI) compatibility of GC microstructures needs to be established to help validate this potential in clinical settings. Here, we present GC microelectrodes and microstructures-fabricated through the carbon micro-electro-mechanical systems process and supported on flexible polymeric substrates-and carry out experimental measurements of induced vibrations, eddy currents, and artifacts. Through induced vibration, induced voltage, and MRI experiments and finite element modeling, we compared the performances of these GC microelectrodes against those of conventional thin-film platinum (Pt) microelectrodes and established that GC microelectrodes demonstrate superior magnetic resonance compatibility over standard metal thin-film microelectrodes. Specifically, we demonstrated that GC microelectrodes experienced no considerable vibration deflection amplitudes and minimal induced currents, while Pt microelectrodes had significantly larger currents. We also showed that because of their low magnetic susceptibility and lower conductivity, the GC microelectrodes caused almost no susceptibility shift artifacts and no eddy-current-induced artifacts compared to Pt microelectrodes. Taken together, the experimental, theoretical, and finite element modeling establish that GC microelectrodes exhibit significant MRI compatibility, hence demonstrating clear clinical advantages over current conventional thin-film materials, further opening avenues for wider adoption of GC microelectrodes in chronic clinical applications.
\end{abstract}

\section{Introduction}

Increasingly, carbon is becoming a compelling material of choice for the micro- and nanofabrication of a variety of micro devices with applications varying from biochemical sensors to microcapacitors and batteries ${ }^{1-3}$. The recent introduction of neural probes consisting of glassy

Correspondence: Sam Kassegne (kassegne@sdsu.edu)

${ }^{1}$ MEMS Research Lab, Department of Mechanical Engineering,College of

Engineering, San Diego State University, 5500 Campanile Drive, San Diego, CA 92182, USA

${ }^{2}$ NSF-ERC Center for Neurotechnology (CNT), Seattle, WA, USA

Full list of author information is available at the end of the article.

These authors contributed equally: Surabhi Nimbalkar, Erwin Fuhrer carbon (GC) microelectrodes microfabricated through carbon micro-electro-mechanical systems (C-MEMS) technology and transferred to flexible polymer substrates has opened up significant opportunities in wearable and implantable carbon devices. This trend will continue as more evidence supporting the superior performance of GC microstructures in applications requiring extended electrical, electrochemical, and mechanical stability under chronic in vivo conditions emerge ${ }^{4}$.

In the meantime, magnetic resonance imaging (MRI) is increasingly being used under pre- and post-surgery conditions for the brain imaging of patients as well as

\section{(c) The Author(s) 2019}

(c) Open Access This article is licensed under a Creative Commons Attribution 4.0 International License, which permits use, sharing, adaptation, distribution and reproduction cc) in any medium or format, as long as you give appropriate credit to the original author(s) and the source, provide a link to the Creative Commons license, and indicate if changes were made. The images or other third party material in this article are included in the article's Creative Commons license, unless indicated otherwise in a credit line to the material. If material is not included in the article's Creative Commons license and your intended use is not permitted by statutory regulation or exceeds the permitted use, you will need to obtain permission directly from the copyright holder. To view a copy of this license, visit http://creativecommons.org/licenses/by/4.0/. 
animal models already implanted with electrocorticography (ECoG) or deep brain stimulation (DBS)type neural probes. These MRI modalities are typically needed to investigate outcomes of DBS or chronic sulcus electrode placement and to evaluate pathological abnormalities related to electrode implantation ${ }^{5}$. They are also used for guiding surgery in chronically implanted microelectrode arrays in inaccessible areas of the cortical sulcus where a magnetization-prepared rapid acquisition with gradient-echo (MPRAGE) sequence is acquired for stereotaxically positioning the array ${ }^{6}$. In addition, the $3 \mathrm{D}$ atlas MRI co-registration method has been used to localize stimulating electrodes of DBS systems in Parkinson's disease patients?

However, there is a significant number of documented cases where neural implants give rise to visible artifacts in MRI images ${ }^{8}$. Specifically, these MR images have shown distortion artifacts due to mismatches in the magnetic susceptibility of electrode materials and brain tissue, leading to inaccurate observations of electrodes in MR images due to hypo or hyper signals at these interfaces ${ }^{7}$. Furthermore, the interaction between conductive materials and radio frequency (RF) fields results in the heating of electrode surfaces, in which the transmitted RF field is absorbed in the electrode material, causing heating ${ }^{9,10}$. An increase in temperature up to $7{ }^{\circ} \mathrm{C}$ due to $\mathrm{RF}$ electrical field induction of current in the leads of electrodes has been reported ${ }^{11}$. In addition, since neural implant materials are usually tested under high magnetic fields (up to $3 \mathrm{~T}$ ), excitation by RF and rapidly switching gradients cause not only imaging artifacts but also eddy currents. The use of MRI, therefore, understandably raises concerns about risks such as heating of the cortex due to induced currents $^{11,12}$ and mechanical movements of electrodes due to gradient-induced vibrations.

In general, while designing cortical electrode arrays compatible with MRI, the hazards to be considered are (1) forces generated due to the interaction between gradient fields and the static $B_{0}$ field with the implanted electrode, (2) induced voltages due to a changing gradient field and RF field and movement of the electrode inside the scanner, (3) heating of the electrode due to RF pulses and electric fields generated by a time-varying magnetic field, and (4) imaging artifacts originating from (a) susceptibility mismatch between the electrode material and surrounding brain tissue and/or (b) $B_{1}$-field distortion due to induction $^{8}$. To avoid or minimize the imaging artifacts, electrode materials with magnetic susceptibility matching that of brain tissue have been introduced, such as printed carbon ink electrodes on organic polymer substrates ${ }^{13}$, titanium-based microelectrodes ${ }^{14}$, silicon microelectrodes for neural recording and stimulations ${ }^{15}$, and carbon nanotube yarn electrodes ${ }^{16}$. However, while these materials have good MRI compatibility, they nonetheless suffer from a lack of long-term electrical and electrochemical stability. In this study, therefore, we build upon the excellent magnetic susceptibility performance of carbon materials and the excellent stability of $\mathrm{GC}^{4}$ and investigate the response of GC microelectrodes to MRI from the perspective of induced voltages, mechanical forces, and vibrations, as well as artifacts. We additionally create a finite element model (FEM) to validate the experimental measurements of induced voltages, currents, and mechanical forces. The aim is, therefore, to extend the application of GC to implantable devices, where other modalities such as live and simultaneous MRI are required for clinical purposes on subjects with implanted neural devices.

\section{Materials and methods \\ Microfabrication of GC microelectrodes}

To investigate and compare the MRI compatibility of GC microelectrodes and thin-film metal microelectrodes, we fabricated probes made of both $\mathrm{GC}$ and $\mathrm{Pt}$ microelectrodes in two sets as shown in Fig. 1. The first set consisted of a ground electrode of an ECoG probe (one with GC and another with $\mathrm{Pt}$ with a rectangular geometry of $2 \mathrm{~cm} \times 1 \mathrm{~cm}$ ) supported on a polymer substrate, while the second set consisted of ring electrodes of $5 \mathrm{~cm}$ outer diameter and $3 \mathrm{~cm}$ internal diameter supported on a silicon wafer. Ground microelectrodes were selected for the MRI study because they represented microelectrodes with the largest surface area in an array of recording and stimulation electrodes in neural probes. The core C-MEMS microfabrication technology that is used for these GC devices supported on polymeric substrates is described in detail elsewhere $^{17}$. Briefly, a SU8-10-negative photoresist (Microchem, Westborough, MA, USA) with a final thickness of $6 \mu \mathrm{m}$ was spin coated, and a ground microelectrode $(2 \mathrm{~cm} \times 1 \mathrm{~cm})$ was patterned. Pyrolysis of the negative resist layer was conducted following protocols described elsewhere $^{18,19}$. In brief, the pyrolysis of the patterned microelectrode array was carried out in a quartz furnace under a vacuum or an inert atmosphere of nitrogen gas with a flow of $50 \mathrm{ml} / \mathrm{min}$ at $1000{ }^{\circ} \mathrm{C}$ for $90 \mathrm{~min}$. The ensuing GC layer was then covered with a layer of nonphotosensitive Durimide 115a and patterned (protocol described elsewhere $)^{4}$. Subsequently, a thicker layer of Durimide 7520 (Fujifilm USA, Inc., Mesa, AZ, USA) was photolithographically patterned to provide a stiffer substrate for the ground microelectrode. This was followed by etching of the silicon dioxide in a buffered hydrofluoric acid bath. For the probes with Pt ground microelectrodes, conventional metal lift-off on a polymer layer was carried out. The details are given in the Supplementary Section (Fig. S1). 

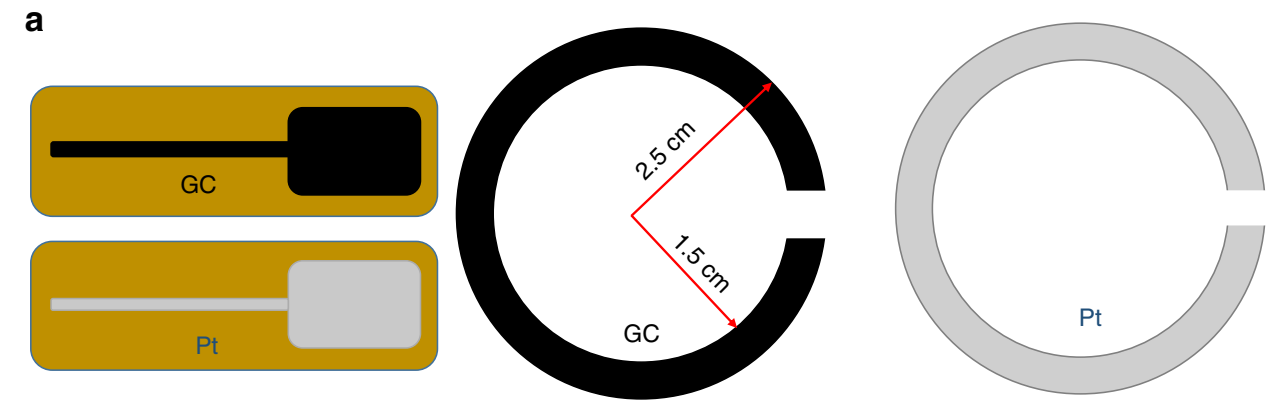

b

1. Spin-coat with negative resist (SU8-10)
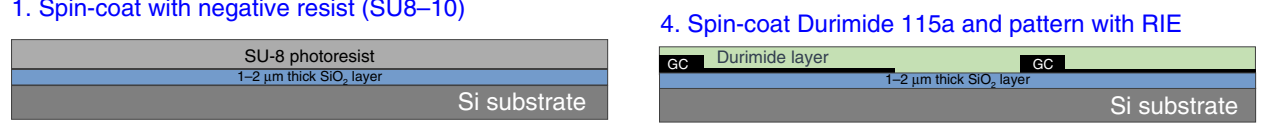

2. Pattern negative resist (photolithography)
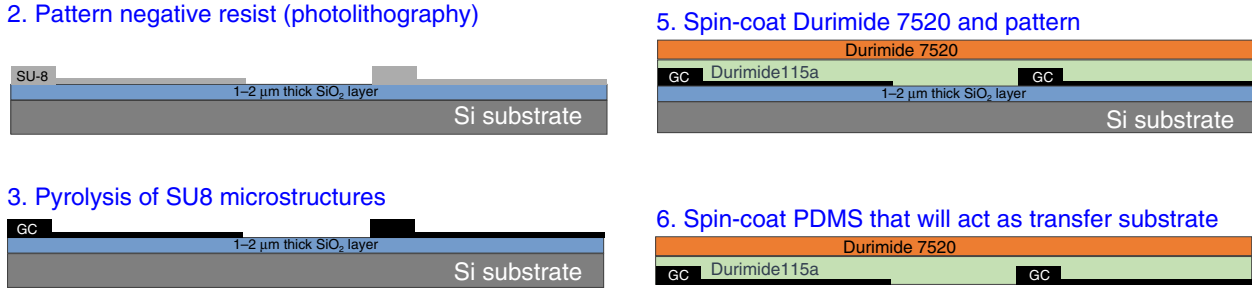

6. Spin-coat PDMS that will act as transfer substrate

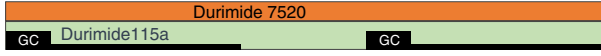

Fig. 1 Test eletrodes for probe characterisation. a Probes with GC and Pt ground microelectrodes with a geometry of $2 \mathrm{~cm} \times 1 \mathrm{~cm}$ supported on a polyimide substrate, and circular $\mathrm{GC}$ and Pt microelectrodes $(\mathrm{OD}=5 \mathrm{~cm}, \mathrm{ID}=3 \mathrm{~cm})$ supported on a silicon substrate. b UV photolithography steps for microfabrication of GC microelectrodes on a flexible substrate

\section{MRI measurements2}

The MR images of GC and Pt microelectrodes of identical dimensions $(2 \mathrm{~cm} \times 1 \mathrm{~cm})$ shown in Fig. 1a were acquired by a Siemens $3 \mathrm{~T}$ Prisma scanner that is commonly used for clinical imaging (Siemens $\mathrm{GmbH}$, Erlangen, Germany). To simulate the brain tissue environment, a phantom was prepared by dissolving phosphate-buffered saline (PBS, $0.01 \mathrm{M})$ in agarose in a glass Petri dish, where the GC and Pt microelectrodes were subsequently immersed. The agarose gel solution was allowed to solidify to avoid the trapping of bubbles. As shown in Fig. 2, the agarose phantom with the microelectrodes was then placed on top of the MRI phantom (solution $\mathrm{N}$ per $1000 \mathrm{~g} \mathrm{H}_{2} \mathrm{O}$ dist.: $3.75 \mathrm{~g}$ $\mathrm{NiSO}_{4} \times 6 \mathrm{H}_{2} \mathrm{O}, 5 \mathrm{~g} \mathrm{NaCl}, 5300 \mathrm{ml}$, Siemens Healthcare $\mathrm{GmbH}$, Germany) to position the microelectrode near the isocenter of the coil. MR images of the PBS-agarose phantom with GC and Pt microelectrodes were collected to test for MRI artifacts (Fig. 2a). The following MR sequences were run with the $3 \mathrm{~T}$ scanner: (i) T1weighted images were acquired using an inversionprepared 3D gradient-echo sequence (MPRAGE) with $0.8 \times 0.8 \times 0.8 \mathrm{~mm}^{3}$ resolution, flip angle $=9^{\circ}$, echo time $(\mathrm{TE})=3.15 \mathrm{~ms}$, repetition time $(\mathrm{TR})=7.7 \mathrm{~ms}$, inversion time $(\mathrm{TI})=900 \mathrm{~ms}$, field of view $(\mathrm{FOV})=200 \times 200 \times$ $179 \mathrm{~mm}^{3}$, and inversion repeat time $=2300 \mathrm{~ms}$; (ii) T2- weighted images were collected using a variable flip angle 3D turbo spin echo (TSE) sequence with $0.9 \times$ $0.9 \times 0.9 \mathrm{~mm}^{3}$ resolution, $\mathrm{TE}=408 \mathrm{~ms}, \mathrm{TR}=3200 \mathrm{~ms}$, FOV $=230 \times 230 \times 173 \mathrm{~mm}^{3}$, and averages $=1.4$; (iii) a $2 \mathrm{D}$ dual echo $B_{0}$-field map was obtained with $3 \times 3 \times$ $2 \mathrm{~mm}^{3}$ resolution, TE $1=4.92 \mathrm{~ms}$, TE $2=7.38 \mathrm{~ms}$, TR $=$ $600 \mathrm{~ms}, \mathrm{FOV}=192 \times 192 \mathrm{~mm}^{2}$, and flip angle $=60^{\circ}$; and (iv) gradient-echo multiband EPI sequence (functional MRI) images were acquired using a multiband echo planar imaging sequence with $2 \times 2 \times 1 \mathrm{~mm}^{3}$ resolution, $\mathrm{TR}=1.02 \mathrm{~s}, \mathrm{TE}=31 \mathrm{~ms}$, and flip angle $=60^{\circ}$.

Furthermore, for a higher spatial resolution, we acquired 3D gradient-echo images (GRE) and $B_{0}$-field maps using an $11.7 \mathrm{~T}$ scanner $(500 \mathrm{MHz}$ Bruker Avance III, Ettlingen, Germany). Due to limited sample space, we trimmed the GC and Pt microelectrodes to quarter pieces and mounted them onto a sample holder that was placed inside the wide-bore magnet, as shown in Fig. 2b. We used a standard 3D Cartesian GRE sequence with $\mathrm{TR}=$ $30 \mathrm{~ms}, \mathrm{TE}=2.02 \mathrm{~ms}, \mathrm{FA}=15^{\circ}$, and an isotropic resolution of $100 \mu \mathrm{m}$. To investigate the effect of $B_{1}$ distortion, the sample was oriented such that the $B_{1}$ vector was orthogonal to the surface of the electrodes ${ }^{20}$. The $B_{0}$-field map was acquired using a predefined integrated protocol in the machine software (ParaVision, FieldMap), which uses two GRE sequences with varying echo times. In this 


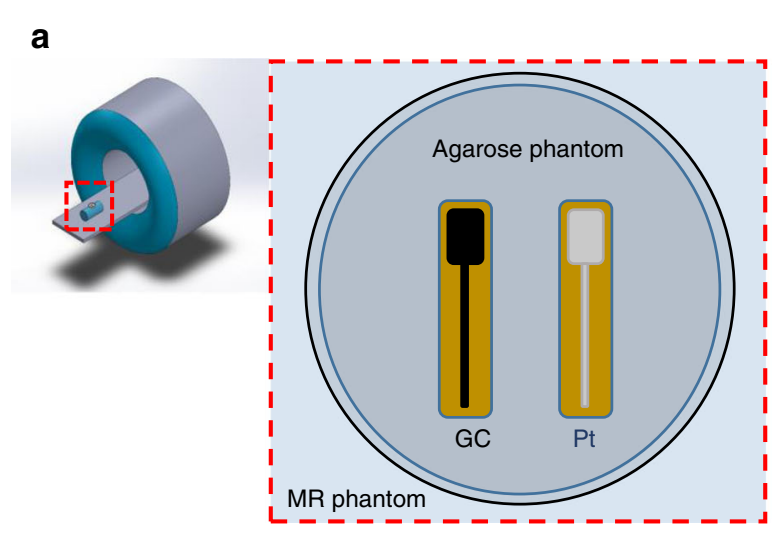

\section{b}

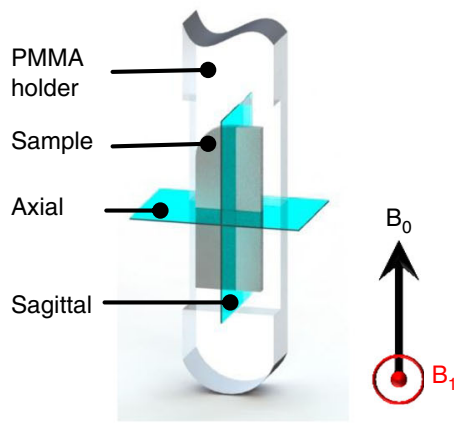

Fig. 2 Arrangement of test probes in the MRI scanner. a 3T Human MRI scanner with glassy carbon and platinum microelectrodes placed on an MRI phantom and GC and Pt cortical microelectrodes immersed in agarose; (b) quarter of a microelectrode specimen mounted in an $11.7 \mathrm{~T}$ scanner

case, the samples were oriented such that the $B_{1}$ vector was parallel to the surface of the microelectrodes to suppress $B_{1}$-field distortions. The $B_{0}$-field map parameters were identical to the GRE sequence except TE1 = $1.675 \mathrm{~ms}$ and TE2 $=26.811 \mathrm{~ms}$.

\section{Finite element modeling of $B_{0}$-field map}

FEM simulations of the effects observed were modeled in COMSOL Multiphysics (COMSOL AB, Sweden) using the $2 \mathrm{~cm} \times 1 \mathrm{~cm}$ rectangular microelectrodes as a domain (Fig. S2). Static magnetic fields ( $B_{0}$ effects) were obtained by solving for the magnetic scalar potential using Gauss' law, the dynamic electromagnetic behavior (gradient effects) by solving for the magnetic vector potential and the current fields using Ampere's and Faraday's laws, and the radio frequency behavior ( $B_{1}$ effects) by solving for the electric field of the Maxwell equations. The simulated materials were Pt $\left(\varepsilon_{\mathrm{r}} \sim=0.735, \chi=279\right.$ p.p.m., $\sigma=9.43 \times$ $\left.10^{6} \mathrm{~S} / \mathrm{m}\right), \mathrm{GC}\left(\varepsilon_{\mathrm{r}} \sim=12.5, X=-1.2\right.$ p.p.m., $\left.\sigma=6803 \mathrm{~S} / \mathrm{m}\right)$, and poly(methyl methacrylate) (PMMA) $\left(\varepsilon_{\mathrm{r}} \sim=2.6, \chi=\right.$ -1.2 p.p.m., $\sigma \sim=0 \mathrm{~S} / \mathrm{m})$ in a water phantom $\left(\varepsilon_{\mathrm{r}} \sim=80, \chi\right.$ $=-9.05$ p.p.m., $\sigma \sim=0 \mathrm{~S} / \mathrm{m})^{21-24}$.

\section{Gradient-induced vibration measurements}

All vibrational experiments were conducted within a $1.5 \mathrm{~T}$ permanent magnet system (Bruker, ICON). A custom-built probe ${ }^{25,26}$ was used to investigate the torque produced by the ground microelectrode when exposed to gradient switching. The main experimental setup is illustrated in Fig. 3. Vibration occurs if conductive structures are placed inside a static magnetic field where an additional time-variable magnetic field featuring a linear gradient is superimposed. In the setup presented here, the produced torque leads to a mechanical deflection that could be precisely measured by reflection of a laser beam using a segmented photodiode.
To acquire the torque produced by different implants, we fixed the microelectrodes onto a PMMA substrate and mounted it to the coil-sample interface. Then, we followed a three-step measurement protocol to determine the torque produced by the implants. The protocol is described in Supplementary Section C.

The mechanical setup illustrated in Fig. 3 is modeled by a harmonic oscillator using Eq. 1.

$$
j \ddot{\theta}+\Gamma \dot{\theta}+\mu \theta=\tau(t),
$$

where $J$ is the moment of inertia, $\Gamma$ is the rotational friction, $\mu$ is the torsion constant, $\tau$ is the external torque, and $\theta$ is the measured deflection angle. We designed the setup to produce the externally applied torque in two ways. The first involved a precisely controlled current $I(t)$ with respect to the amplitude and frequency that was passed through an actuation coil with a determined surface area $A$. This produces the controllable torque shown in Eq. 2. The normal vector of surface $A$ with the vector of $B_{0}$ forms the angle $\alpha$ :

$$
\tau_{1}(t)=I(t) A \sin (\alpha) B_{0} .
$$

The second involves the gradient-switching-produced eddy currents, which lead to a torque $\tau_{2}(t)$ as given by Eq. 3:

$$
\tau_{2}(t)=-\frac{1}{8} \dot{G}_{z} z \pi \sigma t r_{\mathrm{s}}^{4} B_{0} \sin (\alpha) \cos (\alpha),
$$

where $G_{z}$ is the gradient slew rate, $z$ is the position of the implant (center of gravity), $\sigma$ is the electrical conductivity, $t$ is the thickness of the conductive layer, $r_{\mathrm{s}}$ is the largest dimension of the implant, and $\alpha$ is the angle between the normal vector of the implant surface with $B_{0}$ and $G_{z}$ $\left(B_{0} \| G_{z}\right)$. As a control group, we prepared an additional set of samples with (i) a sample exhibiting very high 


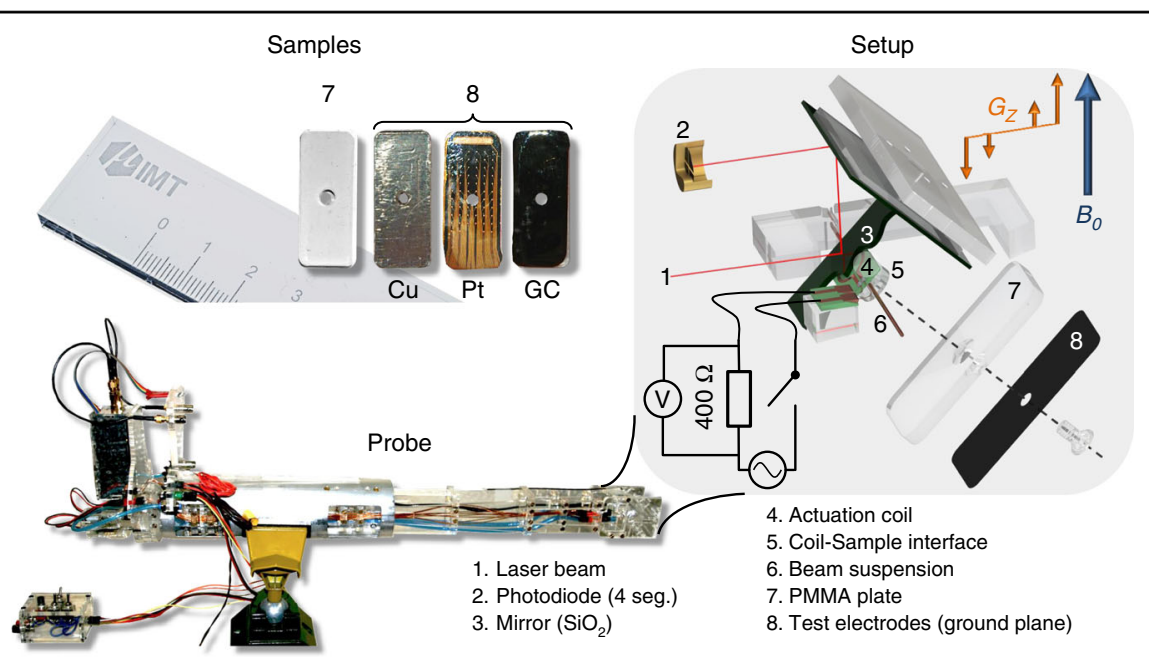

Fig. 3 Details of the experimental setup for the measurement of gradient-induced vibrations in the field of a permanent magnet $1.5 \mathrm{~T}$ MRI system (ICON, Bruker, Germany). Top left: Image (with cm scale bar) of the test samples. Top right: Illustration of the measurement head. The microelectrode (8) is fixed onto a PMMA plate (7) and mounted to a coil-sample interface. The coil-sample interface is suspended on a torsion beam for which the static angular deflection is proportional to the second moment of the cross-sectional area of the beam. The vibration is recorded by measuring the deflection of a laser beam using a segmented photodiode (2). Bottom: Photograph of the complete measurement probe (Cu: copper foil, $40 \mu \mathrm{m}$ thick; Pt: platinum microelectrode, $300 \mathrm{~nm}$ thick; and GC: glassy carbon microelectrode, $2 \mu \mathrm{m}$ thick)

conductivity using a 40- $\mu$ m-thick copper foil (Chomerics CCK-18-101-0200) and (ii) a non-conductive empty PMMA substrate (Fig. 3).

\section{MR-induced current and voltage measurements}

To further investigate the performance difference between GC and Pt, we microfabricated open-loop circular dummy microelectrode structures (outer radius $=2.5 \mathrm{~cm}$ and inner radius $=1.5 \mathrm{~cm}$ ) from these two materials, as shown in Fig. 1. The planar geometry for both GC and Pt was the same, with $\mathrm{Pt} \approx 300 \mathrm{~nm}$ thick and $\mathrm{GC} \approx 2 \mu \mathrm{m}$ thick. For the vibration experiments, we also prepared a highly conductive sample from $\mathrm{Cu}$ tape as a control experiment. The open-loop microstructures enabled direct measurement of the induced voltages and currents, as shown in Fig. 4. First, we measured the resistance $R_{\mathrm{i}}$ of the dummy structure using the four-terminal method (RhodeSchwarz HM 8112-3). In the next step, we measured the induced voltage due to gradient switching in the openloop configuration, followed by a current measurement using a shunt resistor of $1 \Omega$. In these measurements, the surface was placed orthogonal to the gradient field. Direct voltage measurements helped to avoid acoustic coupling.

\section{Results}

\section{MRI measurement}

The imaging artifacts produced in the $3 \mathrm{~T}$ scanner by the GC and Pt microelectrodes were compared in the phantom using clinical MRI sequences. In Fig. 5, the first row shows T1-weighted images taken with the MPRAGE sequence, and the second column shows T2-weighted images taken with the TSE sequence. T1- and T2weighted images are commonly used in clinical sequences and are represented in axial, sagittal, and coronal views in Fig. 5. The darkened rectangular shape on the right side of the phantom artifact shows loss of signal or hyperintense signals (Fig. 5) corresponding to an imaging artifact produced by the Pt microelectrode. High signals or bright spots are observed at the interface of the $\mathrm{Pt}$ microelectrode and the polyimide substrate. Since PBS saline has the same magnetic susceptibility as does brain tissue, it mimics the MR environment of the brain ${ }^{27}$. On the left side of the phantom, an outline of the GC electrode is barely visible (Fig. 5e, f). The Pt electrode sites were $\approx 300 \mathrm{~nm}$ in thickness, but the distortions in $\mathrm{MR}$ images observed near the Pt electrode were more spread throughout the thickness of the agarose phantom $(4 \mathrm{~mm})$ in the axial and sagittal views (Fig. 5a-d). The MRI in Fig. 5 shows that GC microelectrodes $\left(\chi=-1.2\right.$ p.p.m. $\left.{ }^{22}\right)$ have significantly fewer artifacts than do Pt microelectrodes $\left(\chi=279\right.$ p.p.m. $\left.{ }^{27}\right)$. Similarly, images captured with the EPI sequence showed loss of signal at the location of the Pt microelectrode, whereas the GC microelectrode position was not differentiable from the phantom background (Supplementary Fig. S6). There is no visible differentiation between the $B_{0}$-field maps of the $\mathrm{GC}$ and $\mathrm{Pt}$ microelectrodes, as shown in Supplementary Fig. S6b.

\section{High-field MRI}

The results of the $B_{0}$-field measurements and the image experiments conducted in the high-field (11.7 T) magnet are summarized in Fig. 6. The artifacts are mainly due to 


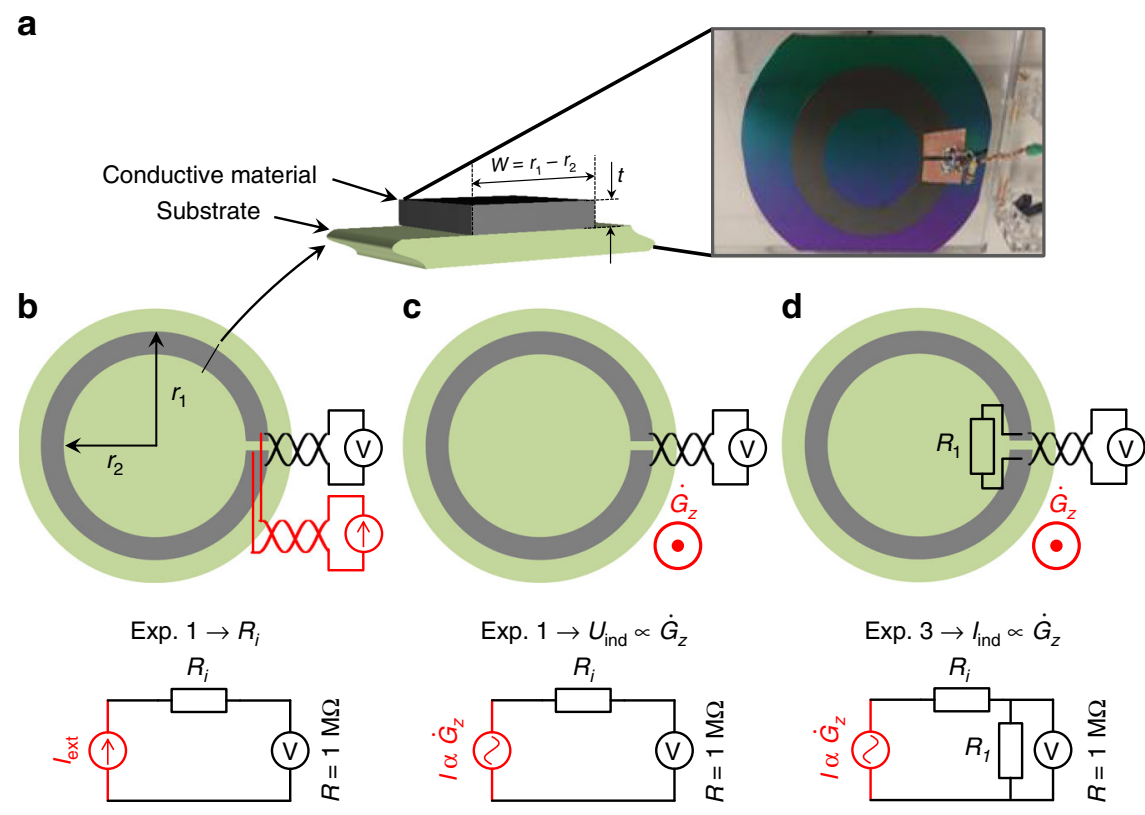

Fig. 4 Experimental setup used to investigate the dependence of microelectrode materials on the induced eddy currents. Preparation of conductive rings using $\mathrm{Cu}$ (thickness $t=40 \mu \mathrm{m}), \mathrm{Pt}(t=300 \mathrm{~nm})$, and $\mathrm{GC}(t=2 \mu \mathrm{m})$. The radii are $r_{1}=2.5 \mathrm{~cm}$ and $r_{2}=1.5 \mathrm{~cm}$. a Sample on a silicon wafer. $\mathbf{b}$ Measurement of $R_{\mathrm{i}}$ by means of four-terminal sensing. $\mathbf{c}$ Measurement of the induced voltage due to gradient switching in open circuit mode. $\mathbf{d}$ Measurement of the induced current using a shunt resistor of $1 \Omega$

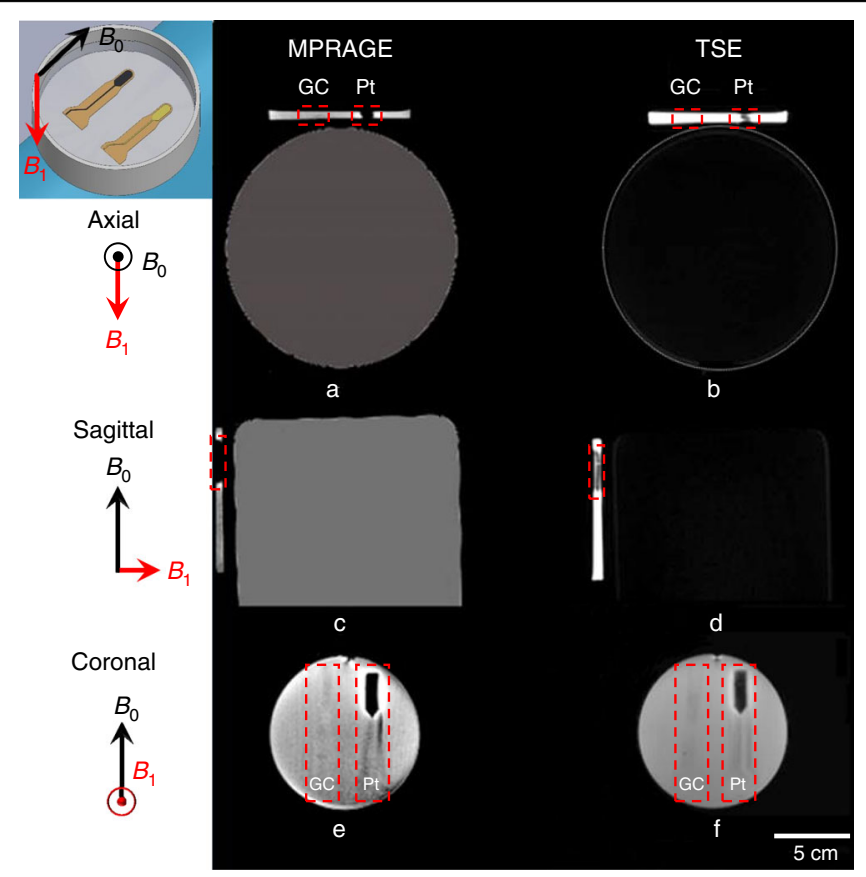

Fig. 5 Comparison of magnetic resonance imaging artifact of GC and Pt microelectrode from T1 -weighted and T2-weighted images captured with MPRAGE and TSE sequences, respectively. Pt electrode placed on right side of the agarose phantom showed darkened rectangular shape along the width of Pt electrode in the axial view as compared to the GC electrode (a, $\mathbf{b})$ for both T1- and T2-weighted images. Similarly, imaging artifact is recorded at Pt electrode along the length in sagittal view of agarose phantom placed on top of MRI phantom (c, d). In the coronal view, bright spots were observed at Pt and polyimide interface, while no artifacts were observed at GC electrode 


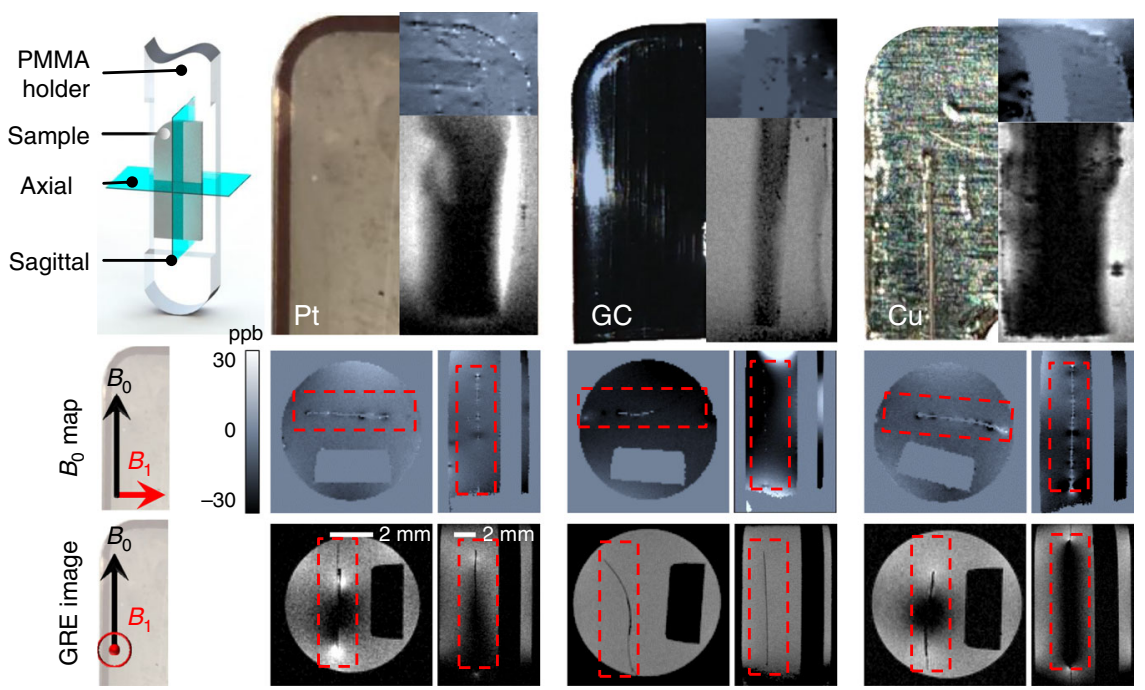

Fig. 6 Results of the $B_{0}$-field maps and GRE images for the Pt and GC microelectrodes and the copper dummy microelectrode. For the $B_{0}$-field map acquisition, the metal samples were oriented such that the $B_{1}$ vector is parallel to the electrode surface and orthogonal for the GRE image sequences. For all samples, no significant field distortion is visible. The color map used for the $B_{0}$-field maps varies from -30 to 30 p.p.b. ( -15 to $15 \mathrm{~Hz}$ ) (blue encoded images). Slight distortions in the range of a few $\mathrm{Hz}$ are visible for the Pt microelectrode. The background field for the GC electrodes shows slight inhomogeneity, but there are no measurable field distortions. Strong $B_{1}$-field distortions are observed for the Pt as well as the Cu dummy samples. This is due to the high conductivity of the materials, which yields considerable eddy currents that counteract the $B_{1}$ field

$B_{1}$-field distortion based on induced eddy currents. This conclusion is supported by the fact that there are no severe $B_{0}$-field distortions in the $B_{0}$-field map, but there are large intensity distortions in the MR images. These intensities are a result of opposing magnetic fields to the $B_{1}$-field and induced eddy currents. Compared to the $\mathrm{Pt}$ microelectrode, the GC microelectrode sample does not show any image distortions because the smaller conductivity inhibits the formation of considerable adverse eddy currents. This is consistent with what was observed with the $3 \mathrm{~T}$ scanner.

\section{Finite element analysis simulations}

From the results obtained and shown in Fig. 7, it can be observed that a reduced susceptibility mismatch $\left(\Delta \chi_{\mathrm{H}_{2} \mathrm{O}-\mathrm{GC}}\right.$ $\left.\sim=0.04 \Delta \chi_{\mathrm{H}_{2} \mathrm{O}-\mathrm{Pt}}\right)$ and drastically reduced area conductivity $\left(\sigma_{\mathrm{tGC}} \sim=0.005 \sigma_{\mathrm{tPt}}\right)$ lead to a significant reduction in artifacts arising from $B_{1}$ and $B_{0}$ inhomogeneity. The relative contribution of these will depend on the imaging sequence used. Similarly, the decreased conductivity led to a reduction in the mechanical response coming from gradient switching, in the same ratio as that of the resistances of each microelectrode. Furthermore, due to the reduced conductivity, the microelectrodes have a smaller interaction with the RF fields, meaning the dielectric heating response will be more accurately described by standard RF/SAR simulations, as optimized for safety. Similarly, any Joule heating coming from current dissipation in the microelectrodes will potentially also be reduced by a similarly proportional factor.
When applying a current/force-generating gradient field to the electrodes $B_{G}=t(\vec{G} \cdot \overrightarrow{\Delta r})$, the behavior on a microelectrode can be split into two separate contributions as follows:

$$
B_{G}=t \vec{G}\left(\overrightarrow{\Delta r}_{\mathrm{CE}-G}+\overrightarrow{\Delta r}_{\mathrm{PE}-\mathrm{CE}}\right),
$$

where $\overrightarrow{\Delta r}_{G-\mathrm{CE}}$ is a function of the distance from the center of the electrode to the center of the gradient's origin and $\overrightarrow{\Delta r}_{\mathrm{PE}-\mathrm{CE}}$ is the distance from a point in the electrode to its center. The first effect was experimentally measured with the resulting torque but can be fully removed by placing the center of the microelectrode at the gradient's origin, whereas the second effect cannot be measured and cannot be removed. Given that it is impossible to precisely position an implant in a patient relative to the magnet, introducing a displacement in the $\mathrm{cm}$ range, the same order of magnitude of the microelectrode itself, the two effects mentioned will be comparable in magnitude.

\section{Artifacts measurement: currents}

Investigating the induced voltages in the ring structures allowed for the examination of the gradient field interaction without acoustic noise distortions. Similar to the microelectrode design, the GC and PT samples had the same geometry, except that the thicknesses of the layers were different ( $\mathrm{Pt} \approx 300 \mathrm{~nm}$ and $\mathrm{GC} \approx 2 \mu \mathrm{m}$ ). The resistances of the test structures were $R_{\mathrm{Pt}}=25 \Omega$ for the Pt-based microstructure and $R_{\mathrm{GC}}=280 \Omega$ for the GC 


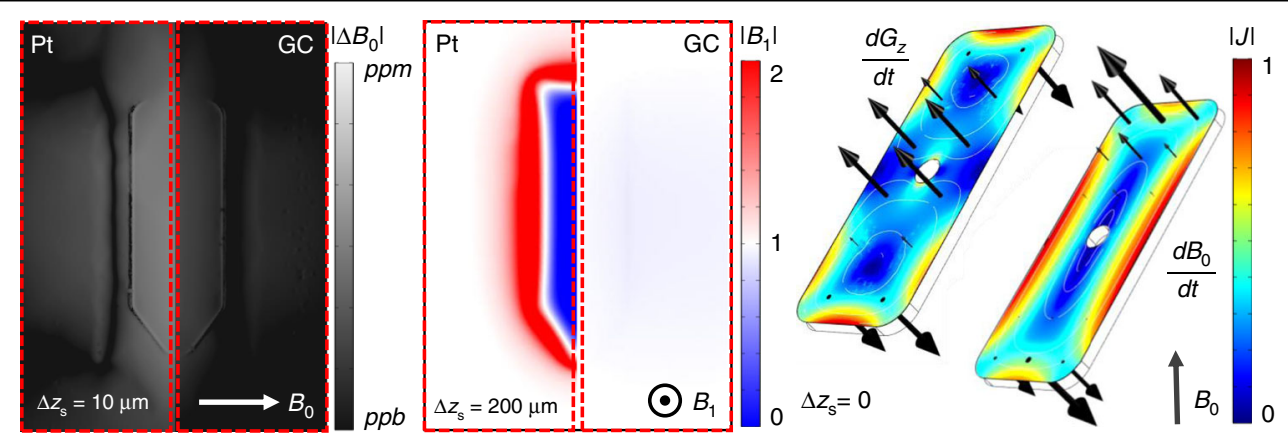

Fig. 7 Comparison of the simulated behavior for the $2 \mathrm{~cm} \times 1 \mathrm{~cm}$ rectangular GC and Pt ground microelectrodes. On the left, one can see the $B_{0}$ relative-inhomogeneity map, at the center, the normalized $B_{1}$ inhomogeneity map and, on the right, the induced currents and forces generated by each effect of a unitary gradient's slew rate, as described above. The results are for a water phantom in a $1.5 \mathrm{~T}$ field, shown for varying distances to the electrode surface, $\Delta z_{s}$, where the effects are at magnitudes relevant to the experiment: 1 p.p.m. for inhomogeneity at $\Delta z_{s}=10 \mu \mathrm{m}$ (left image), hundreds of micrometers for a slice thickness (middle image), and current dissipation on the surface of the electrode (right image)

microstructure, and $R_{\mathrm{Cu}} \leq 0.006 \Omega$ for the $\mathrm{Cu}$ tape. As expected, the induced voltage depended only on the surface through which the magnetic flux changed. The theoretical value presented in Fig. 8 is computed using Eq. 5:

$$
U[V]=\pi\left(r_{1}^{2}-r_{2}^{2}\right) \dot{G}_{z} z
$$

The gradient slew rate in this experiment was $2046.6 \mathrm{~T} /$ $\mathrm{m} / \mathrm{s}$, which leads to the theoretical voltage value displayed in Fig. 8, showing that the measured induced voltage in the rings is in good agreement with the theoretical value (solid line). The measurements with the short end are also presented in the same figure to highlight the amplitude of erroneous induced voltage in the measurement lines. The induced currents measured with a $1 \Omega$ resistor are also given in the same figure, indicating that the current in GC is at least a factor of 10 less than that of the Pt sample. Notably, the current induced in the GC was below the limit of detection (LOD) when using a resistor of $1 \Omega$.

\section{Vibration measurements}

In Fig. 9, the experimental results are displayed against the theoretical values. The acoustic coupling was measured using empty PMMA plates, which allowed us to determine the LOD. This means that samples with conductive structures are beyond the LOD if their vibration amplitude is not distinguishable from the non-conductive PMMA plates. This is the case for both the Pt and GC microelectrode samples. The sample with copper foil $(\mathrm{Cu})$, in contrast, led to strong vibrational amplitudes of more than two orders of magnitude above the LOD. With this sample, the common mode vibration is clearly detectable where the vibration approaches zero at the gradient center. The acoustic vibration, on the other hand, is strongest inside the MR scanner $(140 \mathrm{~mm})$, which leads to the best LOD being achieved at $\sim 30-40 \mathrm{~mm}$. At this point, the gradient slew rate is strongest, whereas the acoustic coupling is minimal.
Using theoretical analysis, we can estimate how much the torque of the implants is beyond the LOD. The quality of this analysis is benchmarked by comparing the predicted and experimental values of the $\mathrm{Cu}$ sample.

Interestingly, the platinum microelectrode appears to be at the LOD, whereas the GC microelectrode has an approximately 40 -fold weaker response (see Fig. 9b). In contrast, the copper foil results in a response three orders of magnitude larger than that of the tested electrodes. We want to emphasize that due to the linearity of electromagnetism, the derived value of torque per unit slew rate may serve as a figure of merit for an implant, which allows a simple derivation of the worst-case torque at any MR scanner. As an example, we may take the $\mathrm{Cu}$ foil with a TPSR $=4 \frac{\mu \mathrm{Nm}}{\mathrm{T} \times \mathrm{T} / \mathrm{s}}$. Then, in a $3 \mathrm{~T}$ human MR scanner with a slew rate of max. $300 \mathrm{~T} / \mathrm{m} / \mathrm{s}$ and largest dimension of $0.3 \mathrm{~m}$ (e.g., in the $z$ direction), the maximum expected torque is evaluated as:

$$
\begin{aligned}
\tau_{\max } & =\operatorname{TPSR} \times B_{0} \times G_{z} \times z \\
& =4 \frac{\mu \mathrm{Nm}}{\mathrm{T} \times \mathrm{T} / \mathrm{s}} \times 3 \mathrm{~T} \times 300 \mathrm{~T} / \mathrm{m} / \mathrm{s} \times 0.3 \mathrm{~m}=1080 \mu \mathrm{Nm} .
\end{aligned}
$$

According to $\mathrm{ASTM}^{28}$, critical torque is reached when the MR-induced torque is larger than the gravitationalinduced torque. Gravitational-induced torque can be computed using the weight $(m)$ of an object multiplied by its longest dimension $(l)$. Hence, for the $\mathrm{Cu}$ foil, the gravitational torque $\tau_{\mathrm{g}}$ is:

$$
\begin{aligned}
\tau_{\mathrm{g}}=m g l= & 8960 \mathrm{~kg} / \mathrm{m}^{3} \times(0.04 \times 25 \times 11) 10^{-9} \mathrm{~m}^{3} \\
& \times 9.81 \mathrm{~m} / \mathrm{s}^{2} \times 25 \times 10^{-3} \mathrm{~m} \\
& =24.2 \mu \mathrm{Nm} \ll 1080 \mu \mathrm{Nm} .
\end{aligned}
$$

Because $\tau_{\max }$ » $\tau_{\mathrm{g}}$, the $\mathrm{Cu}$ foil is clearly MR incompatible. On the other hand, the upper limit for the MR-induced 

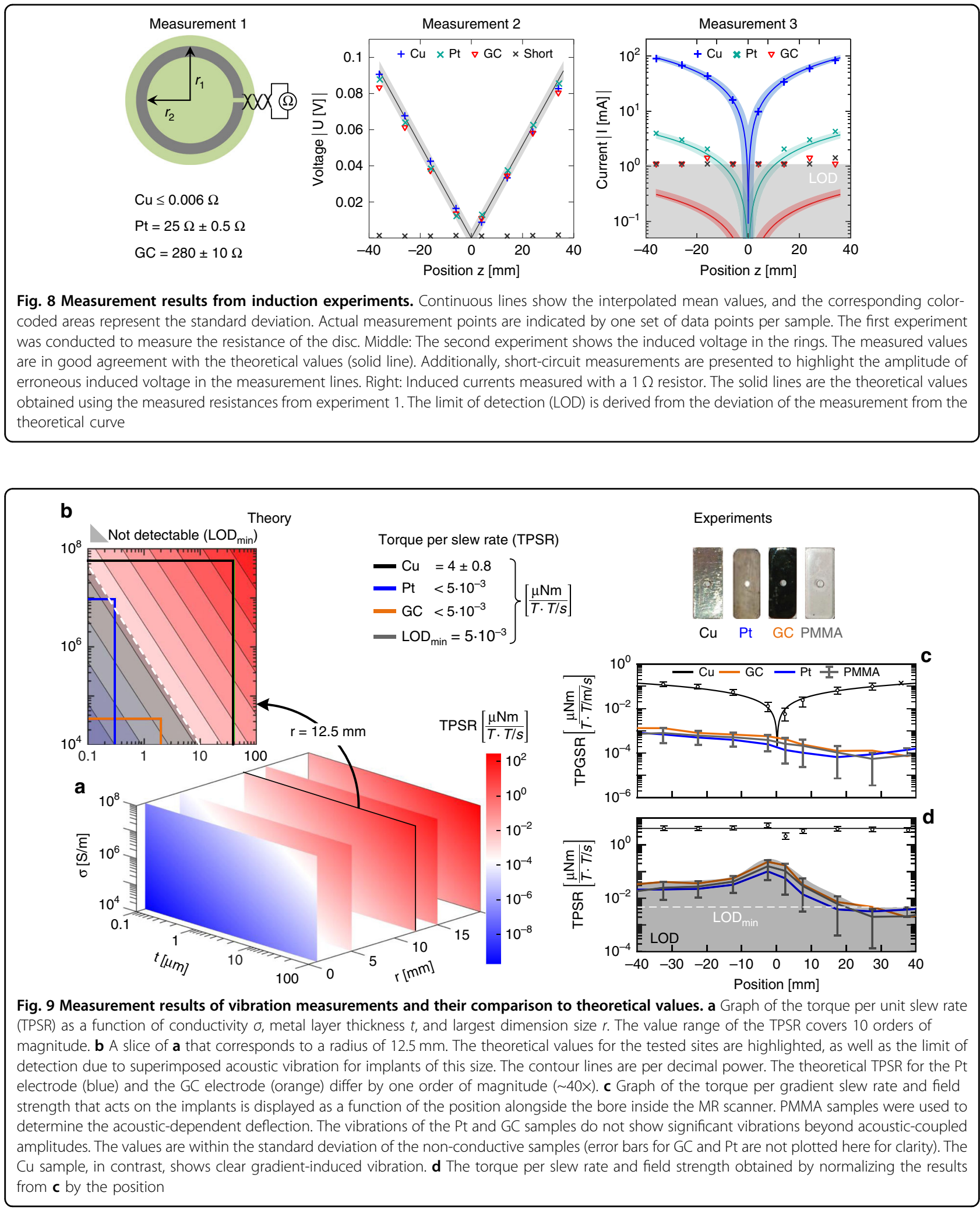

torques of the $\mathrm{Pt}$ and $\mathrm{GC}$ microelectrodes are in the range of $5 \times 10^{-3} \times 3 \mathrm{~T} \times 300 \mathrm{~T} / \mathrm{m} / \mathrm{s}=4.5 \mu \mathrm{Nm}$, which is safe.

\section{Discussion/conclusions}

In this work, the MR interactions of GC- and Pt-based microelectrodes were investigated with respect to their 
adverse effects in producing MR artifacts and gradientinduced vibrations. The specific aim of the MR characterization was to test whether GC microelectrodes used for DBS or the less-invasive intracortical recording (ECoG) produce any artifacts while mapping functional or morphological information of the brain with MR. With regard to gradient-induced vibrations, calculations show that the induced eddy currents are much larger for the $\mathrm{Pt}$ microelectrodes than for the GC microelectrodes. However, for both types of microelectrodes, the measurable forces were below the detection limit. In our experimental scanner, the gradient field slew rates are up to a factor of ten larger than in comparable human MRI machines; hence, the expected forces with the presented microelectrodes should remain within a non-hazardous range even in human scanners with up to $7 \mathrm{~T}$. We want to emphasize, however, that the gradient-induced vibration scales to the power of four with the radius of the implants; thus, for larger electrodes, the positive effect of the smaller conductance of GC will be advantageous in obtaining electrode designs that are not prone to vibration induction. We also emphasize the introduction of a new figure of merit that is independent of field strength and gradient slew rate. It provides an important tool for comparing the tendency of different implants to vibrate and enables the straightforward computation for the worst-case torque of an implant for any field strength and slew rate.

MR artifacts, on the other hand, in contrast to vibrations, depend on the conductivity as well as the magnetic susceptibility of the applied material. In summary, our findings show that the larger magnetic susceptibility of platinum does not produce considerable field distortions, especially because of the small volume of the thin-film electrodes. Both GC microelectrodes showed field distortions with <30 p.p.b. at even $11.7 \mathrm{~T}$, and from this viewpoint, both microelectrode types may even allow for use within field-sensitive applications such as localized spectroscopy in human MR machines operating with smaller static magnetic fields $(\leq 3 \mathrm{~T})$. On the other hand, the Pt microelectrode shows considerable $B_{1}$-field distortions due to RF-induced eddy currents, which lead to large position-dependent obscuration or hyperintensity. The GC microelectrode is clearly superior with respect to this type of artifact, showing no measurable distortion of the image. The reason is also the much smaller electrical conductivity of GC. It should be noted here that the geometries of these microelectrodes affect their resistance (and hence conductivity), as the resistance $R$ is a function of length $L$ and cross-sectional area $A$ (i.e., $R=\rho L / A$ or $R$ $=L /(\sigma A)$ ), suggesting that the contribution of geometry alone to the resistance $R$ is $\sim 6.6 \times$ for Pt compared to GC (since the thickness of $\mathrm{Pt}=300 \mathrm{~nm}$ and of $\mathrm{GC}=2 \mu \mathrm{m}$ ). However, the conductivity $(\sigma)$ of $\mathrm{Pt}$ is $9.43 \times 10^{6} \mathrm{~S} / \mathrm{m}$, which is almost $1400 \times$ that of GC $(6803 \mathrm{~S} / \mathrm{m})$, in effect making the resistance $R$ of the $\mathrm{Pt}$ microelectrode $\sim 1 /$ 200th that of GC. Therefore, despite the smaller thickness, Pt microelectrodes experienced higher induced currents, as demonstrated here. For eddy currents, on the other hand, where the RF resistance is more relevant, length plays a larger role than does thickness due to skin effects; hence, the resistance of Pt and GC microelectrodes will be dominated by their inherent conductivities $^{29}$.

Therefore, we submit that GC microelectrodes demonstrate superior behavior with respect to MR safety compared to Pt-based electrodes. There is strong indication that this statement will be further corroborated by an analysis of RF-induced heating, which was not conduced in this investigation. One large source of RF-induced heating is RF-induced eddy currents; the same currents lead to large MR image artifacts only in the Pt microelectrode and not in the GC microelectrode. We anticipate that the introduced figure of merit will be of great significance as an implant-specific value for labeling torque-related interactions.

Taken together, this study demonstrated that (i) GC microelectrodes experienced no considerable vibration deflection amplitudes and minimally induced currents (below the LOD), while Pt microelectrodes had considerably larger currents, (ii) GC microelectrodes had almost no susceptibility shift artifacts compared to Pt microelectrodes because GC has 1/20th the magnetic susceptibility of Pt and (iii) GC microelectrodes had no eddy-current-induced artifacts, unlike the Pt microelectrodes, mainly because of the lower conductivity of GC $(\sim 1 / 1000$ th that of $\mathrm{Pt})$ that inhibits the formation of considerable eddy currents. Since GC has recently been demonstrated to have a compelling advantage over other materials for neural stimulation, recordings, and electrochemical sensing of neurotransmitters through voltammetry ${ }^{4,19}$, this MRI compatibility validated in this study offers an additional advantage for long-term in vivo use in clinical settings.

\section{Acknowledgements \\ This material is based on research work supported by the Center for Neurotechnology (CNT), a National Science Foundation Engineering Research Center (EEC-1028725), and on a detection modality established within the framework of the German Excellence Initiative under grant number EXC 1086 (BrainLinks-BrainTools). E.F. and J.G.K. would also like to acknowledge support from the European Union's Future and Emerging Technologies Framework (H2020-FETOPEN-1-2016-2017-737043-TISUMR).}

\section{Author details}

${ }^{1}$ MEMS Research Lab, Department of Mechanical Engineering,College of Engineering, San Diego State University, 5500 Campanile Drive, San Diego, CA 92182, USA. ${ }^{2}$ NSF-ERC Center for Neurotechnology (CNT), Seattle, WA, USA. ${ }^{3}$ Institute of Microstructure Technology - Karlsruhe Institute of Technology, Hermann-von-Helmholtz-Platz, 76344 Eggenstein-Leopoldshafen, Germany.

${ }^{4}$ Magnetic Resonance Imaging Lab, San Diego State University, San Diego, CA 92182, USA 


\section{Conflict of interest}

The authors declare that they have no conflict of interest.

Supplementary information accompanies this paper at https://doi.org/ 10.1038/s41378-019-0106-x.

Received: 15 March 2019 Revised: 28 June 2019 Accepted: 27 August 2019 Published online: 18 November 2019

\section{References}

1. Ranganathan, S., McCreey, R., Majji, S. M. \& Madou, M. Photoresist-derived carbon for microelectromechanical systems and electrochemical applications. J. Electrochem Soc. 147, 277-282 (2000).

2. Beidaghi, M., Chen, W. \& Wang, C. Electrochemically activated carbon microelectrode arrays for electrochemical micro-capacitors. J. Power Sources 196, 2403-2409 (2011)

3. Wang, C. et al. Carbon-MEMS architecture for 3D microbatteries. In: Urey, H., ElFatatry, A. (eds), Proc. SPIE-Photonics Europe, Strasbourg, France, vol 5455 (April 2004). https://doi.org/10.1117/12.548755.

4. Nimbalkar, S. et al. Ultra-capacitive carbon neural probe allows simultaneous long-term electrical stimulations and high-resolution neurotransmitter detection. Nat. Sci. Rep. 8, 6958 (2018).

5. Arantes, PaulaR. et al. Performing functional magnetic resonance imaging in patients with Parkinson's disease treated with deep brain stimulation. Mov. Disord. 21, 1154-1162 (2006).

6. Scherberger, $\mathrm{H}$. et al. Magnetic resonance image-guided implantation of chronic recording electrodes in the macaque intraparietal sulcus. J. Neurosci. Methods 130, 1-8 (2003).

7. Yelnik, J. érôme et al. Localization of stimulating electrodes in patients with Parkinson disease by using a three-dimensional atlas-magnetic resonance imaging coregistration method. J. Neurosurg. 99, 89-99 (2003).

8. Erhardt, J. B. et al. Korvink. Should patients with brain implants undergo MRI? J. Neural Eng. 15, 041002 (2018).

9. Shellock, FrankG. Radiofrequency energy-induced heating during MR procedures: a review. J. Magn. Reson. Imaging 12, 30-36 (2000).

10. Dempsey, MaryF. \& Condon, Barrie Thermal injuries associated with MRI. Clin. Radiol. 56, 457-465 (2001).

11. Finelli, D. A. et al. MR imaging-related heating of deep brain stimulation electrodes: in vitro study. Am. J. Neuroradiol. 23, 1795-1802 (2002).

12. Rezai, A. R. et al. Neurostimulation systems for deep brain stimulation: In vitro evaluation of magnetic resonance imaging-related heating at 1.5 tesla. J. Magn. Reson. Imaging 15, 241-250 (2002).

13. Bonmassar, G., Fujimoto, K. \& Golby, A. J. PTFOS: flexible and absorbable intracranial electrodes for magnetic resonance imaging. PLOS ONE 7, e41187 (2012).
14. Yoon, $\mathrm{H}$. et al. Development of titanium needle probes for neural recording and evaluation of magnetic resonance imaging artifacts. J. Nanotechnol. Eng. Med. 1, 011004 (2010).

15. Martínez-Santiesteban, F. M., Scott, D. S., Douglas, C. N. \& David, J. A. Magnetic resonance compatibility of multichannel silicon microelectrode systems for neural recording and stimulation: design criteria, tests, and recommendations. IEEE Trans. Biomed. Eng. 53, 547-558 (2006).

16. Jiang, C. Q., Hao, H. W. \& Li, L. M. Artifact properties of carbon nanotube yarn electrode in magnetic resonance imaging. J. Neural Eng. 10, 026013 (2013).

17. Kassegne, S. et al. Electrical impedance, electrochemistry, mechanical stiffness, and hardness tunability in glassy carbon MEMS $\mu \mathrm{ECOG}$ electrodes. Microelectron. Eng. 133, 36-44 (2015).

18. Vomero, M. et al. Novel pattern transfer technique for mounting glassy carbon microelectrodes on polymeric flexible substrates. J. Micromech. Microeng. 26, 36-44 (2016).

19. Vomero, M. et al. Highly stable glassy carbon interfaces for long-term neural stimulation and low-noise recording of brain activity. Nat. Sci. Rep. 7, 40332 (2017).

20. Camacho, C. R., Plewes, D. B. \& Henkelman, R. M. Nonsusceptibility artifacts due to metallic objects in MR imaging. J. Magn. Reson. Imaging 5, 75-88 (1995)

21. Windt, D. L. et al. Optical constants for thin films of Ti, $\mathrm{Zr}, \mathrm{Nb}, \mathrm{Mo}, \mathrm{Ru}, \mathrm{Rh}, \mathrm{Pd}$, Ag, Hf, Ta, W, Re, Ir, Os, Pt, and Au from $24 \AA$ to $1216 \AA$. Appl. Opt. 27, 246-278 (1988).

22. Noto, K., Saito, K., Kawamura, K. \& Tsuzuku, T. Diamagnetism of glassy carbons. Jpn. J. Appl. Phys. 14, 480 (1975).

23. Raymond, A. S. Principles of Physics 2nd edn, 602 (Saunders College Pub., Fort Worth, TX; London, 1998).

24. Wapler, M. C. et al. Magnetic properties of materials for MR engineering, micro-MR and beyond. J. Magn. Reson. 242, 233-242 (2014).

25. Fuhrer, E., Jouda, M., Gruschke, O. G. \& Korvink, J. G. Optical gauge head to evaluate gradient field induced vibrations of conductive structures during MRI. In: Glaglia, R. G. (ed), 2017 International Conference on Electromagnetics in Advanced Applications (ICEAA), 1555-1558 (IEEE, 2017).

26. Fuhrer, E., Jouda, M., Klein, C. O. \& Korvink, J. G. Gradient-induced mechanical vibration of neural interfaces during MRI. In: Pan, X. (ed) IEEE Trans. Biomed. Eng. (Early Access) (2019). https://doi.org/10.1109/TBME.2019.2923693.

27. Zhao, Siyuan. et al. Graphene encapsulated copper microwires as highly MRI compatible neural electrodes. Nano Lett. 16, 7731-7738 (2016).

28. ASTM. ASTM F2213-06: Standard Test Method for Measurement of Magnetically Induced Torque on Passive Implants in the Magnetic Resonance Imaging (ASTM International, 2016).

29. Johnson, H. \& Graham, M. High-Speed Signal propagation Advanced Black Magic 3rd edn, ISBN 978-0-13-084408-8, 58-78 (Prentice-Hall, 2003). 